\title{
IoT application for vehicles identification using the Optical Fiber Sensors and Wireless Sensor Network
}

\author{
Hacen Khlaifi ${ }^{1, a}$, Amira Zrelli1,2,b and Tahar Ezzedine ${ }^{1, c}$ \\ ${ }^{1}$ Communication System Laboratory, National Engineering School of Tunis, University Tunis El Manar, Tunisia. \\ ${ }^{2}$ Faculty of sciences of Gabes, University of Gabes, Tunisia \\ aHacen.Khlaifi@enit.utm.tn bAmira.zrelli@enit.utm.tncTaharezzedine.enit@gmail.com
}

\begin{abstract}
Due to the renewed variation in government and political systems inside and outside countries, and with the high tariffs at borders, the latter have become an outlet for terrorism and smugglers. Therefore, each country seeks to develop its own protection system, and the technologies used in these systems vary according to the severity and the importance of the installations to be protected, it is found that some of them are expensive and unnecessary, but other have good and variable levels of efficiency. Consequently, the idea of designing a surveillance system that can monitor and control access becomes indispensable. In the same context, this work is of crucial strategic and geopolitical importance. It combines pre-existing alarm and monitoring methods and revolutionary Internet of Things (IoT) application products, of which Wireless Sensor Networks (WSN) and Optical Fiber Sensors (OFS) are part of this application. This article presents the distribution of wireless radar nodes accompanying with a Bragg fiber sensor to identify each rolling intruder incoming the zone to be monitored, from the determination of its speed, weight and wheelbase distance.
\end{abstract}

INDEX TERMS Classification; Wireless Sensors Networks ; Fiber Bragg Grating; Fluorescence Intensity Ratio ; Pressure; Speed; Wheelbase distance; Weight; Vehicle.

\section{INTRODUCTION}

The IoT term "Internet of Things" is omnipresent: it is found everywhere, such as the industry, military, civil, agricultural, etc.. Communities have been organized to regulate, standardize, and promote the Internet of Things. Researchers and entrepreneurs have looked at the IoT to set up and promote the applications they offer. According to the International Telecommunication Union (ITU), the IoT is a global information system infrastructure that allows the provision of advanced services by interconnecting components (physical and virtual) based on existing technologies, interoperable information, and communication [1]. There are a number of areas in which IoT finds applications. No areas of daily life will escape this over the next decade. The IoT finds its full realization in industry. The spike in technological development is giving industry a favor with the German approach to controlling the market and offering a cutting edge manufacturing solution called Industry 4.0 [2]. The research presented in the article [3] shows the first integration of an
IoT system based on optical fiber sensors in stator bars of an electric generator to monitor the operation of the system by tracking the temperature variation. C.Xiaojun et al. [4] solved the problem of air pollution via an IoT prediction system based on a WSN. On the other hand, for the agricultural sector, J.C. Zhao et al. [5] proposed an IoT control application using radio frequency identification (RFID) technology RFID is a method for storing and retrieving data remotely using markers called "RFID Tag." Concerning the military domain, a research work presented in [6] [7] [8] shows an IoT application consisting of the surveillance of military zones through a WSN design. In the civil field, the article [9] [10] presented an application that consists of monitoring bridges by predicting the catastrophes that affect them.

Political clairvoyance and military vigilance aim to establish its authority over its domain and control access to its domain; hence, the idea of designing a system to control access to it. The work that we present is of major strategic 
and geopolitical importance. It will combine pre-existing alarm and monitoring methods and revolutionary products from IoT, the commonly used WSN, and the fiber optic sensor, which is used much less often in this field. We will present a system that we will lay out schematically: wireless sensors associated with an optical fiber sensor to detect each rolling intruder entering the area to be monitored.

In this article, an IoT application architecture is proposed, which is based on optical fiber and WSN sensors, making it possible to detect any intrusive vehicle in the area to be monitored. A calculation system consists of determining the weight of the vehicle using the pressure detected by the Bragg fiber sensor, as well as the wheelbase distance through the speed picked up by the radar, in order to determine the type of vehicle passing through the system. . In the first section, we present the problem and the requirements of our system and the phenomenology of the targets by choosing the types of sensors to be used in our design and will develop the operating principle of the Bragg fiber sensor and the FIR.

In the second section, we describe the architecture and algorithm of the application. Finally, we discuss the results.

\section{PROPOSED SYSTEM FOR VEHICLE DETECTION}

\section{A. PROBLEMATIC AND REQUIREMENT}

With the proliferation of violations of national and international laws and the ramification of intra-and interstate transgressions, security is becoming a major issue that preoccupies the authorities. Historically, the borders between states have been drawn in a rather thorny area, which is difficult to access and control. Boundaries between states are therefore fertile grounds for crime and smuggling, which are very harmful to the security and safety of the state.

Border control is an element of prosperity and progress; hence, it is important. The identification of everything entering and leaving the national domain is extensive. While the air and maritime domains seem to be perfectly mastered, the land domain often escapes the eye of the state. Through our work, we will try to allow the state to have control over its entire terrestrial domain, especially over its external terminals, but also within the state itself. To curb this scourge, which affects all combined states, researchers are constantly designing identification and surveillance systems.
In this work, we only focused on rolling objects crossing the area in question. Therefore, our system is also concerned with access control through the identification of any incoming target.

\section{B. PHENOMENOLOGY OF TARGET AND SENSORS CHOOSING}

Our application requires a rolling target, all of which are in constant contact with the ground at any time during the event. The detector that will be installed must be buried underground, at a depth that does not affect its sensitivity to the parameters it is supposed to collect. Because each object in contact with the ground exerts a weight force on it, and knowing that car manufacturers have divided vehicles according to their weight into several categories (see table1), we will be able to calculate the weight accordingly. Unfortunately, this parameter does not seem to be sufficient to determine the category to which the vehicle belongs. In fact, the calculated weight will include the weight of the unladen vehicle added to the load carried (passengers, luggage, goods, etc.).

Once the vehicle is placed in its appropriate class, we know its empty weight. The difference between the empty and detected weights can be interpreted according to context. We need a second parameter to complete the classification operation of the vehicle in question.

Among the characteristics that can inform us about the category in which a vehicle is classified, these are its dimensions. Vehicles in the same category could have completely contrasting dimensions, except for one dimension, which will only be the wheelbase distance (Figure 1) (the distance between the front wheels and the rear wheels). The latter is standardized. Although it can vary from vehicle to vehicle, the variation within the same class is marginal.

We have advocated the use of fiber optic sensors to accomplish this task of detect, calculate, and determine these details. This is because fiber optic sensors are excessively sensitive, allowing the exact calculation of pressure and weight. As the vehicle passes through the fiber optic sensors necessarily over 2 times, they will be able to determine the time interval separating the two times. We physically translated this interval as the time distance wheelbase. To complete the equation, we call on another datum, which is speed. We will entrust this speed calculation mission for wireless radar. In addition to the speed that the main parameter wanted for our work, the radar sensor also makes it possible to determine two other 
parameters in parallel, namely, the acceleration and the degree of orientation of the moving object. This arsenal of sensors, fiber optics, and wireless radars will enlighten us on the two parameters (weight and wheelbase distance), which, taken together, will determine the category of the intruder vehicle.

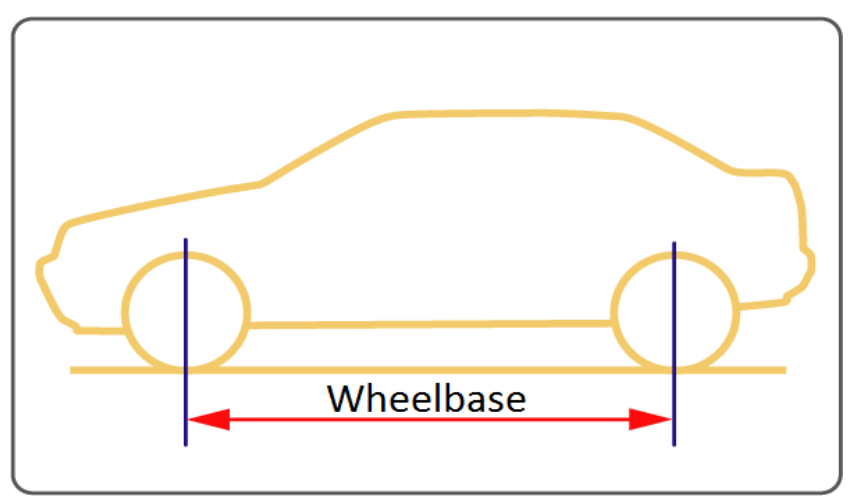

FIGURE 1. Wheelbase distance

III. Vehicle classification via OFS and Radar

Optical fiber sensors (OFS) are currently considered to be the most interesting and promising sensing technologies. Compared with electronic and traditional sensors, OFS has many advantages such as reduced weight and volume, high precision, immunity against electromagnetic interference, resistance and sensitivity to perturbations, electrical isolation, and low transmission losses [17]. Several parameters, such as temperature, humidity, pressure, displacement, strain, and material corrosion were monitored to evaluate the safety of the structure. Indeed, OFS is sensitive to various parameters (strain, pressure, and shape) and optical refractive index and mode conversion [18]. Radar is considered an important and innovative sensor technology that has already been successfully applied to many driver assistance functions. Radar applications have been widened to numerous civilian applications such as traffic control, border monitoring, remote sensing, car surveillance, and collision avoidance $[19,20]$.

\section{A. VEHICLE WEIGHT DETERMINATION \\ 1. TEMPERATURE AND PRESSURE DISTINGUISHED USING FBG AND FIR}

- $\quad F I R$

Changes in the energy levels of the doped ions in the fiber excited by a pumped light source can generate fluorescence intensity (FIR) depending on certain parameters relating to the materials of construction of the fiber, the level of energy and the method of 'excitation. This intensity of fluorescence alone shows its dependence on temperature variation in the vicinity [11]. Therefore, the temperature change can be inferred by calculating the change in FIR, so the numerical relationship between the temperature changes by the FIR is as follows [11]:

$\Delta F I R=C_{F I R}^{T} \times \Delta T_{F I R}$

$C_{F I R}^{T}:$ FIR and temperature dependency constants.

\section{- $\quad F B G$}

The experiments carried out on the FBG deduced a relation between the variation of the reflected wavelength and the variation of the refractive index $\eta[12]$.

$\lambda_{B}=2 \eta \Lambda$

$\lambda_{B}$ : reflected wavelength in the FBG, $\Lambda$ : grating period, $\eta$ : refractive index of the core.

Research conducted in $[13,14,16]$ shows that the wavelength injected into the FBG changes depending on several parameters, such as temperature and pressure.

$\frac{\Delta \lambda_{B}}{\lambda_{B}}=\Delta \lambda_{B}^{T}+\Delta \lambda_{B}^{p}=K_{F B G}^{T} * \Delta T_{F B G}+K_{F B G}^{p} * \Delta p_{F B G}$

$$
\left(\begin{array}{c}
\Delta \lambda_{B}^{T} \\
\Delta \lambda_{B}^{p}
\end{array}\right)=\lambda_{B}\left(\begin{array}{ll}
K_{F B G}^{T} & \Delta T_{F B G} \\
K_{F B G}^{p} & \Delta p_{F B G}
\end{array}\right)
$$

\section{- FBG and FIR concatenation}

Based on the research conducted in the article [15], it is not easy to distinguish between the variation of temperature and the variation of the strain as well as the variation in temperature and pressure. We know that a physical temperature variation causes strain, pressure, and humidity variation, and vice versa. In our case, we cannot conclude whether there is a pressure variation resulting from the atmospheric temperature variation, or a result of mechanical stress on the fiber. Therefore, the idea presented in this manuscript is to specify which causes the other temperature or pressure; then, the FIR technology allows us to pursue the temperature variation around the mechanism in points practically far from the mechanical stress. This method helps us to solve the problem of continuing climatic heating independently of other variations in pressure or strain; hence, the distinction between the variation of temperature and pressure in the system through the concatenation of FIR and FBG technology.

$\Delta T_{F I R}=\Delta F I R / C_{F I R}^{T}$ 
$\Delta T_{F B G}=\Delta T_{F I R}$

$$
\left(\begin{array}{c}
\Delta \lambda_{B}^{T} \\
\Delta \lambda_{B}^{p}
\end{array}\right)=\lambda_{B}\left(\begin{array}{cc}
K_{F B G}^{T} & \frac{\Delta F I R}{C_{F I R}^{T}} \\
K_{F B G}^{p} & \Delta p_{F B G}
\end{array}\right)
$$

\section{WEIGHT DETERMINATION BASED ON PRESSURE RECOVERED BY OFS}

A vehicle crosses the fiber with four wheels: in the 1st time by its front wheels and in the 2 nd time by its rear wheels (Figure 2). The fiber then calculates the pressure at each of the two times. Indeed, according to the model standardized by the manufacturers, the architecture of the vehicles imposes the distribution of their weight uniformly over the entire chassis; thus, half of the weight is supported by the two front wheels, while the 2nd half is supported by the two rear wheels (Figure 3).

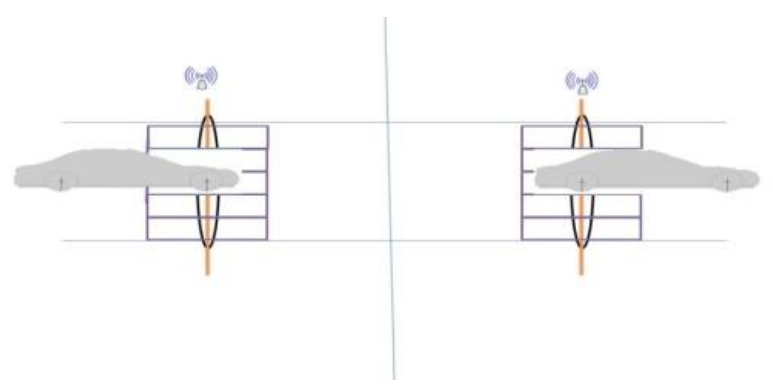

Figure 2. Cases of vehicle contact with the fiber

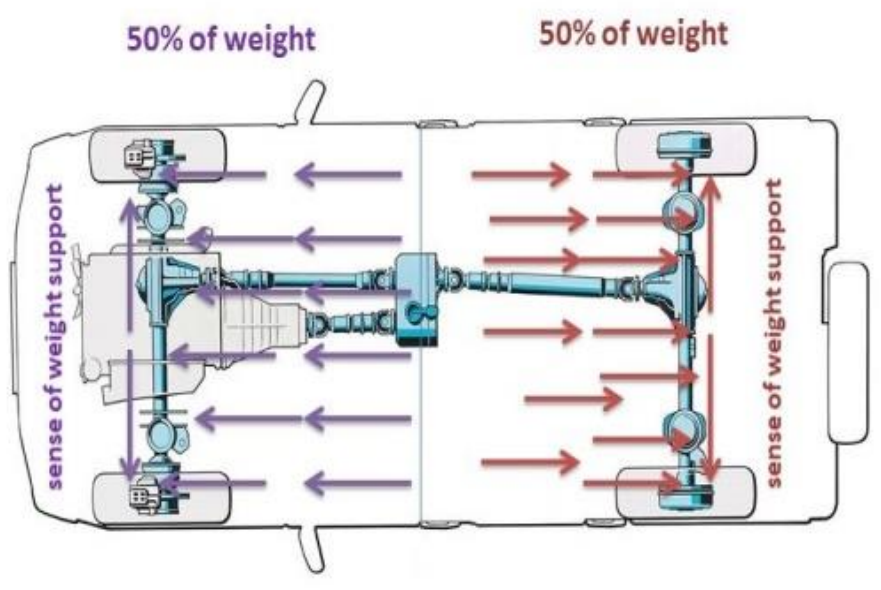

Figure 3. Weight distribution on the vehicle frame

Note that the calculated weight will include the weight of the driver, and possibly other passengers and objects on board the vehicle. Therefore, a simple addition operation will allow the calculation of the total weight of the vehicle. As a first step, we must determine the weight of the vehicle based on this parameter of pressure, while admitting that this parameter will not be sufficient to identify the class of the vehicle. However, this step remains a prerequisite for this process. We recall that the calculation of the pressure here will obey the classical formula (8): the pressure is worth the division of the weight by the section. The only obstacle is that the section parameter is missing.

Pressure $=\frac{\text { weight }}{\text { Section }}$

The section is unknown to us because it comes from the dimensions of the wheels of the vehicle, which are specific to each type of vehicle. To counter this setback, we propose to preliminarily force the section through the implementation of rigid metal plates that are superimposed on the optical fiber. The dimensions of the plates were defined in advance and were uniform (Figure 4).

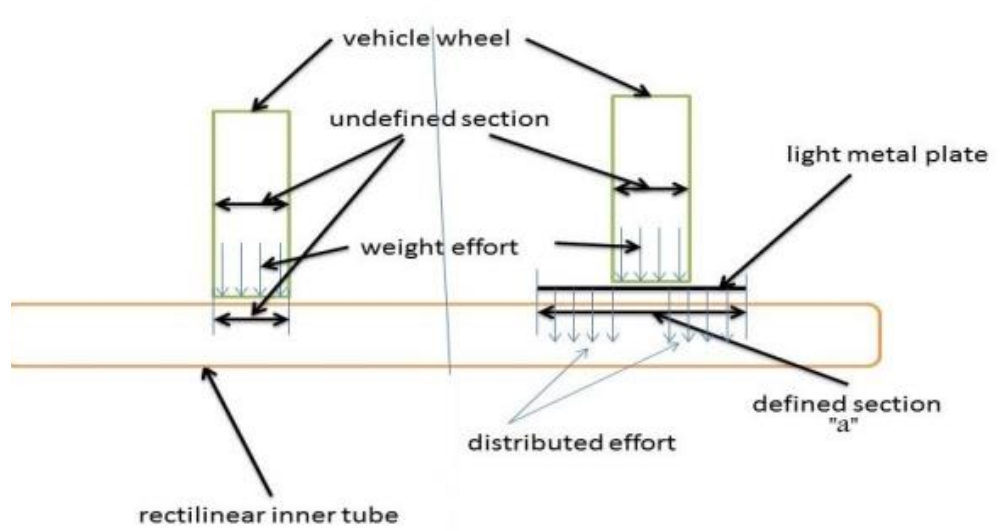

Figure 4. Section determination

When the wheel comes into contact with the metal plate totally or partially, we will obtain the section "a" ("a" is the total section in the case of Bicycles). The total section, when the vehicle crosses by its two front wheels, is equal to $2 *$ a, because in the case of vehicles with parallel supports (parallel wheels), the fiber can instantly detect the number of contacts at each event.

\section{B. VEHICLE WHEELBASE DETERMINATION}

Regarding the determination of the wheelbase $d_{w b}$, when the two rear wheels in turn touch the inner tube, it can be calculated using the speed $V$ detected by the radar and the time lapse $t$ that separates the 1 st contact of the front wheels and the 2nd contact of the rear wheels with the fiber (Figure 2).

Therefore, from equation (9), we can determine the value of the wheelbase distance

$$
d_{w b}=V * t
$$

\section{DESIGN AND SYSTEM ALGORITHM}


Our system, based on fiber-optic sensors, must be completely concealed. Therefore, it is deployed under the ground. The system will be surrounded by a layer of polystyrene with a specific density to keep it free from any wear related to oxidation and shearing. Unfortunately, polystyrene has a relatively long cooling time, which explains the use of the inner tube as a complement (Figure $6,7)$. Our system will be centered in an air chamber, the pressure of which will be calculated to allow our system to respond immediately to the slightest contact. The inner tube allows us to minimize refresh time. The system will be capped by a series of metal plates (Figure 6) whose capital role expected by their installation is to determine the section essential for the calculation of the weight, as indicated previously. These plates are limited by a shoulder, as shown in Figure 7, to prevent slippage of the metal plate and a clearance that allows the metal plate to flatten out whenever pressure from a rolling object occurs. The bending of the plate illustrated in Figure 7 has the function of reducing the cooling time. Therefore, it has a function similar to that of the air chamber.

In short, the system will have the following architecture: an optical fiber-based system hidden under the ground, interconnected to wireless radar nodes (Figure 5,6) whose operating algorithm (Figure 8 ) is designated as follows:

Before the vehicle enters the coverage area of the fiber optic system, the radar collects certain defining information, including speed, acceleration, and degree of orientation. These data were sent to the computer system. On the first contact of the rolling object with the fiber optic system, the latter will be actuated to determine the pressure exerted on it. On the 2 nd contact with the rolling object, the fiber optic system determines the time interval between the two contacts. Therefore, he will be able to calculate the weight and the wheelbase distance using the equations already mentioned above. From the wheelbase distance found, the computer system will match this value and the vehicle that corresponds to it in the database, an extract of which is presented in Table. Once the type of vehicle has been demystified, we can move on to the next step, which is only to determine the difference between the weight indicated on the table and the weight found by the system. The difference indicates the additional load on the board.

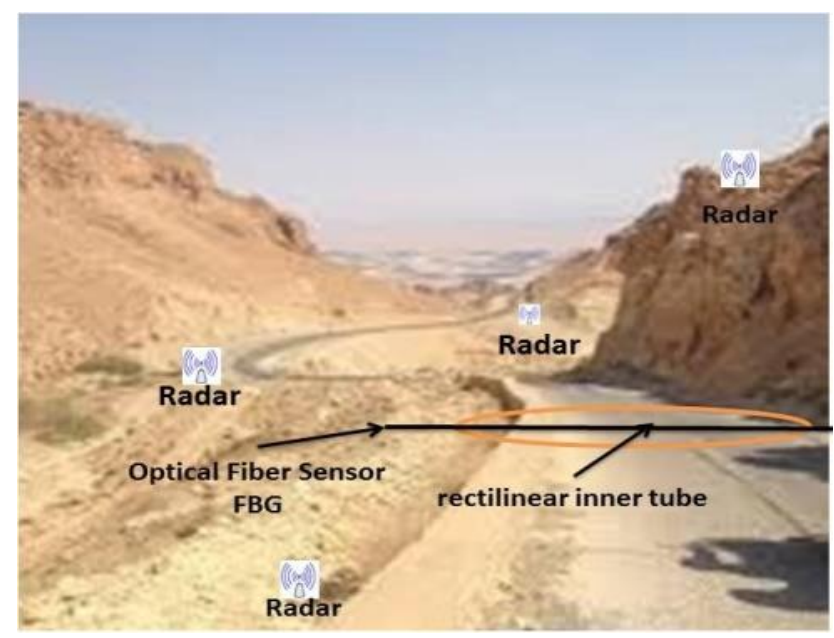

Figure 5. Real design

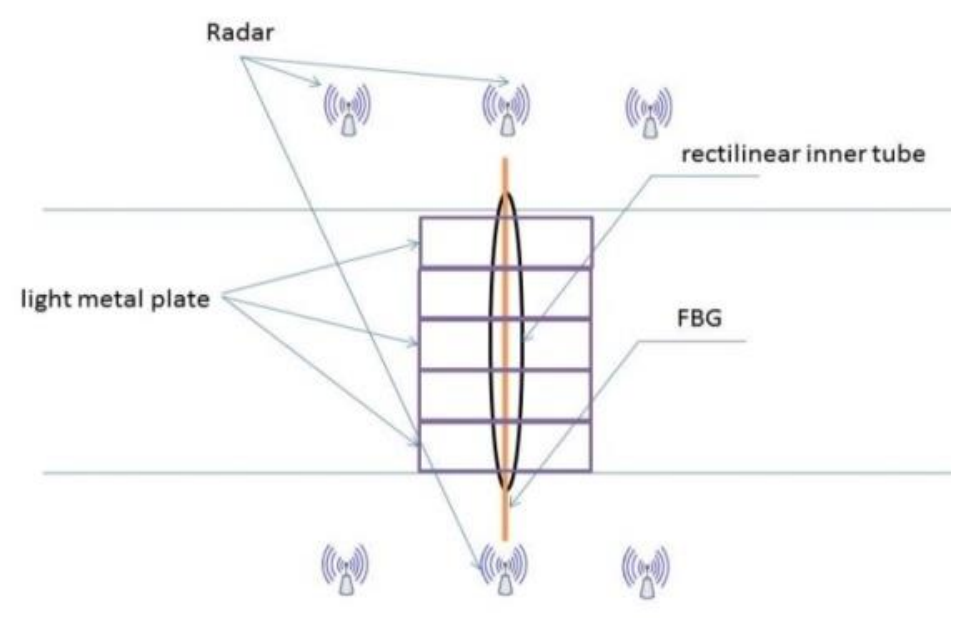

Figure 6. Model design

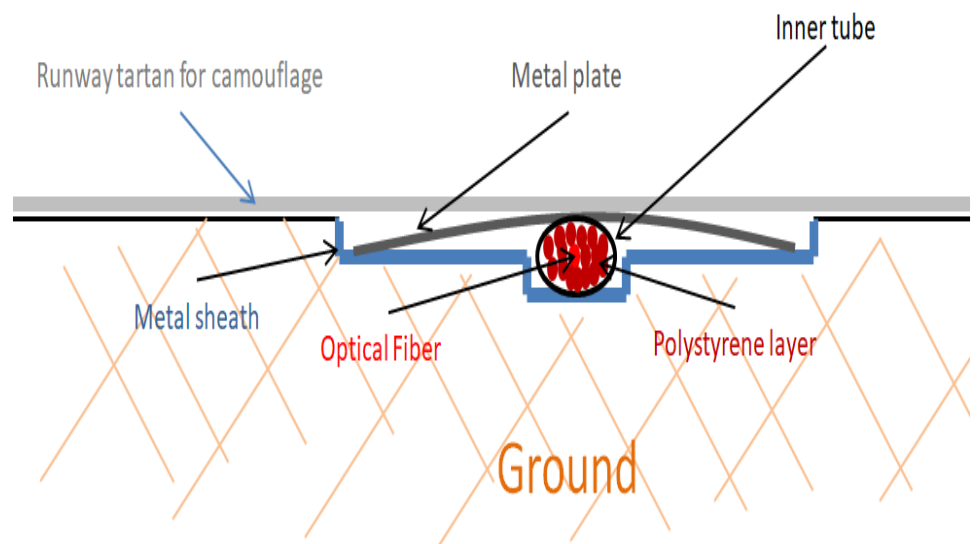

Figure 7. Deployment of the weight detection system based on the optical fiber sensor 


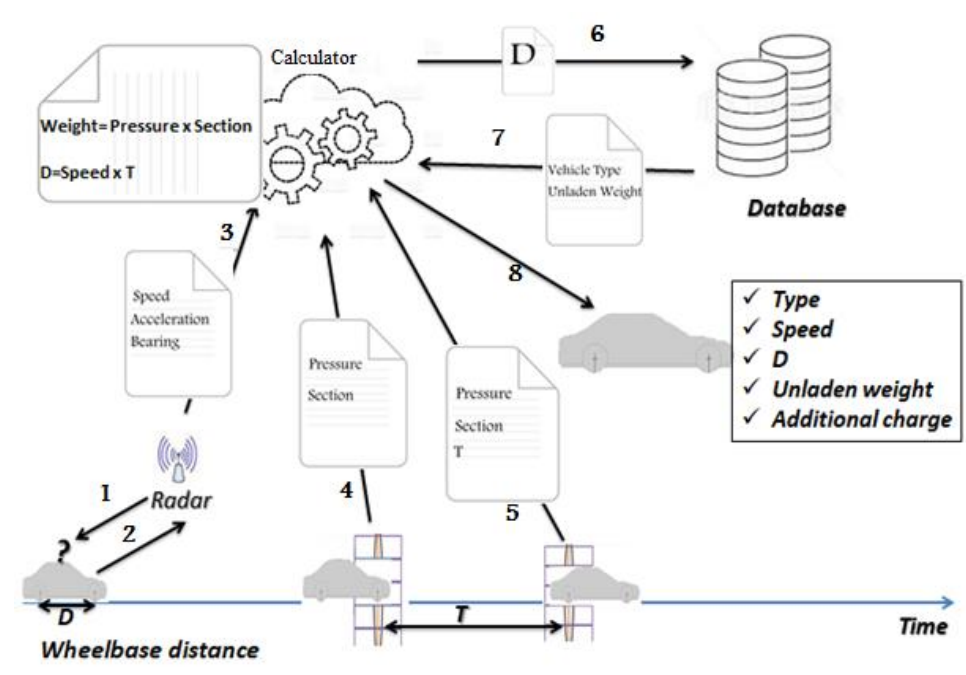

Figure 8. Algorithm

TABLE 1

AN EXTRACT FROM THE DATABASE

\begin{tabular}{llll}
\hline & & Unladen weight & $\begin{array}{l}\text { Wheelbase } \\
\text { distance }\end{array}$ \\
\hline & A1 & $750 \mathrm{Kg}-800 \mathrm{~kg}$ & $2 \mathrm{~m}$ \\
Type A & A2 & $800-850 \mathrm{~kg}$ & $2.3 \mathrm{~m}$ \\
& A3 & $850-900 \mathrm{~kg}$ & $2.4 \mathrm{~m}$ \\
& B1 & $1400-1500 \mathrm{Kg}$ & $2.6 \mathrm{~m}$ \\
Type B & B2 & $1500-1600 \mathrm{~kg}$ & $2.7 \mathrm{~m}$ \\
& B3 & $1700-1800 \mathrm{~kg}$ & $2.9 \mathrm{~m}$ \\
Type C & & 16000 & $4.2 \mathrm{~m}$ \\
Type D & & 12000 & Chain vehicle \\
\hline
\end{tabular}

\section{RESULTS AND DISCUSSIONS}

The curve presented in figure 9 shows the linearity of the pressure compared to the wavelength with an average error rate of $0.05 \mathrm{~kg} / \mathrm{cm}^{2}$, this error rate, being tiny, will not have an impact on calculating the weight from the detected pressure. Figure 10 shows the relationship between the sensitivity detected by the fiber and the actual pressure exerted on the apparent surface of the system. From the results obtained in the same figure10, the difference is almost $-4.77 \mathrm{~dB}$ which reflects the division of the pressure values detected via the fiber by 3 in order to determine the values of the real pressures exerted on the outer surface of the system. The results presented in Figures 11, 12, and 13 are the results of four experiments carried out on four types of vehicles: A, B, C, and D (Table 1).

Figures 11 and 12 show an experiment carried out in three tests, where each test corresponds to a different speed. The peaks shown in the figures denote the moment when the system is triggered by the weight exerted, the sensitivity value of which explains the amount of pressure exerted by the weight. The distance between the peaks refers to the wheelbase interval, which results in the calculation of the vehicle's wheelbase distance.

As shown in Figure 11, we performed three tests on a type A vehicle (weight of $820 \mathrm{~kg}$ and additional load of $200 \mathrm{~kg}$ (130 kg forward and $70 \mathrm{~kg}$ back) with a wheelbase distance of $2.3 \mathrm{~m}$ ) for three speeds. The green curve corresponds to the speed $63.72 \mathrm{~km} / \mathrm{h}$ which is determined by the radar (Figure 11) shows 2 peaks separated by a time lapse of 129 ms. This lapse of time allows us to determine the wheelbase distance from the speed which is $2.283 \mathrm{~m}$ (the error rate is $0.016 \mathrm{~m}$ ) which is far from overlapping with the other values of other vehicles in the base of data.The first peak indicates a sensitivity value of $8.01 \mathrm{~dB}$ which corresponds to a pressure value of $6.31 \mathrm{~kg} / \mathrm{cm}^{2}$ at the level of the fiber and according to the curve of figure 10 the pressure exerted on the external surface of the system is equal to $2.13 \mathrm{~kg} /$ $\mathrm{cm}^{2}$, this value indicates the real value of the pressure exerted by the vehicle.

The weight value which coincides with the pressure value "2.13 kg $/ \mathrm{cm}^{2}$ " is $527 \mathrm{~kg}$ (error rate equal to $13 \mathrm{~kg}$ ). Regarding the second peak, the calculated weight value, which corresponds to a sensitivity of 7.52, is 470.7 (error rate $=9.219 \mathrm{~kg}$ ). The same applies to the other curves (Figure 11 and Figure 12).

Figure 13 presents two curves designating the tests on the two other types of vehicles, C and D. The blue curve concerns the test on the heavy vehicle of type $\mathrm{C}$ (weight: $1600+70 \mathrm{~kg}$, wheelbase distance: $4.2 \mathrm{~m}$, speed: $32.5 \mathrm{~km} / \mathrm{h}$ ), which shows a fairly wide distance between the two peaks, which explains a fairly large wheelbase distance of is $463 \mathrm{~ms}$, which corresponds to a wheelbase distance of $4.19 \mathrm{~m}$ (rate error of $0.01 \mathrm{~m}$ ), and the two peak levels give us a weight value of $16083 \mathrm{~kg}$ (error rate is $13 \mathrm{~kg}$ ). The orange curve concerns the D-type vehicle (weight: $12000 \mathrm{~kg}$, undetermined wheelbase distance: chain vehicle, speed: $34.8 \mathrm{~km} / \mathrm{h}$ ), the shape of the curve and the number of peaks, as well as the levels of sensitivity between the peaks explains the presence of a chain and six secondary wheels between the two main wheels. Here, the vehicle weight is the sum of two weights calculated from the two sensitivity values presented by the two main peaks, and this sum is equal to $12011.4 \mathrm{~kg}$ (error rate equal to $11.4 \mathrm{~kg}$ ).

To measure the performance of our system, we used two parameters: the response time and the refresh time. 
Response time: is the time lapse between the first moment of contact of the object with the system and the pressure peak returned by the system.

Refresh time: is the time lapse between the peak returned by the system and $1 / 3$ of the total pressure exerted by the object itself. The choice of the value $1 / 3$ is not accidental: either a vehicle weighing $800 \mathrm{~kg}$ (400 front / 400 rear), the maximum load supported by the front wheels of the vehicle is $400 \mathrm{~kg}$ depending on the vehicle manufacturer, which is equivalent to $1 / 3$ of the total vehicle load in this case. After reaching the 1st peak, capped at a pressure exerted by a weight of $800 \mathrm{~kg}$, during damping, the last value returned just after the 2nd peak must not be greater than or equal to the value of the 2 nd peak, so that the 2 nd peak is also detectable, even with the naked eye.

According to the results presented in (Figure 11, 12, and 13), the value of the response time was determined to be $0.008 \mathrm{~s}$ and the refresh time value was $0.042 \mathrm{~s}$ (Table2).

TABLE 2

EXPERIMENTAL VALUES

\begin{tabular}{cc}
\hline \multicolumn{2}{c}{ EXPERIMENTAL VALUES } \\
\hline Parameter & value \\
\hline $\begin{array}{c}\text { response time } \\
\left(\boldsymbol{t}_{\boldsymbol{r} p}\right)\end{array}$ & $0.008 \mathrm{~s}$ \\
$\begin{array}{c}\text { refresh time } \\
\left(\boldsymbol{t}_{\boldsymbol{r}}\right)\end{array}$ & $0.042 \mathrm{~s}$ \\
\hline
\end{tabular}

Tow exceptional scenarios could arise:

1. 2 vehicles traveling in the same direction at different speeds.

2. A vehicle traveling at $110 \mathrm{~km} / \mathrm{h} .110 \mathrm{~km} / \mathrm{h}$ being the maximum speed authorized on Tunisian roads.

Regarding the 1 st case, the radars installed, having the ability to detect more than one moving object at a time, will therefore return the speed of each.

Regarding the 2nd case (vehicle traveling at a speed of 110 $\mathrm{km} / \mathrm{h}$ ), we chose to experiment with the vehicle with the shortest wheelbase distance, defined by the manufacturers at $1.3 \mathrm{~m}$ which corresponds to $0.042 \mathrm{~s}$. For the system to be efficient, the refresh time must be strictly less than ( wheelbase time-response time). As it happens:

Response time $=0.008<$ refresh time $=0.019<$ wheelbase time - response time $=0.034$
We conclude that the inequality is valid, and therefore the system is functional even in the most extreme situations, where, according to these two cases, it is possible to install this system in urban road traffic.

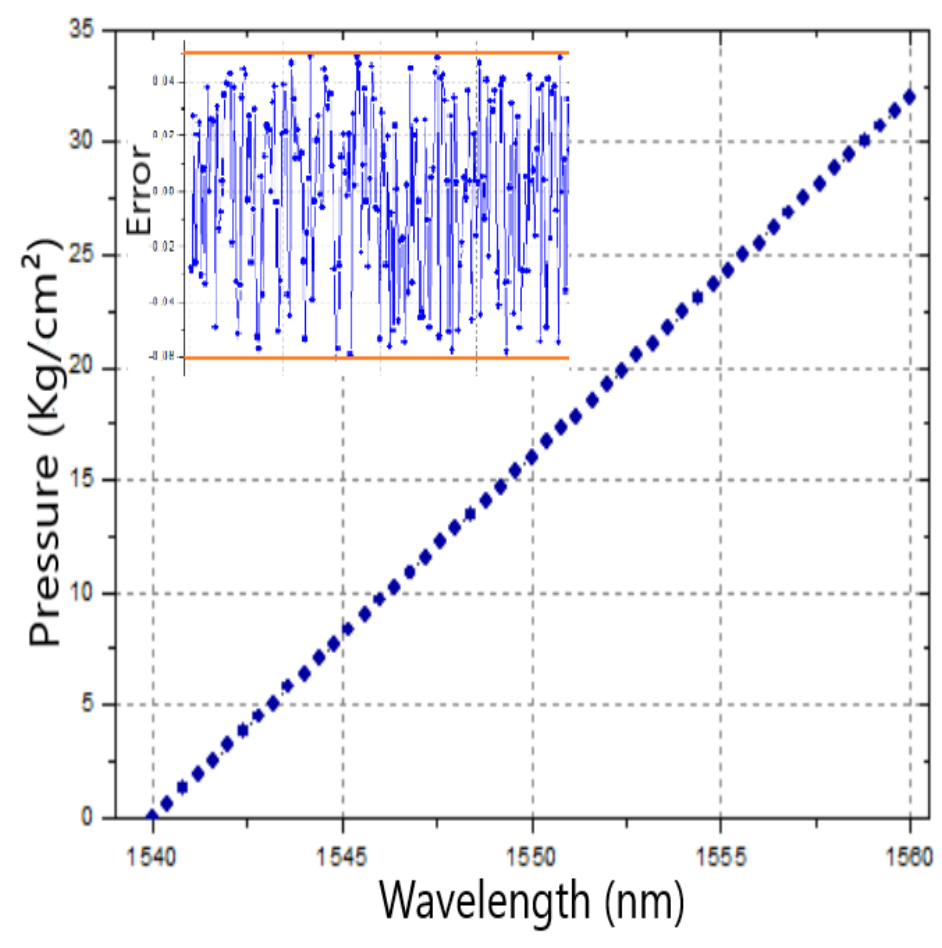

Figure 9. Pressure and wavelength dependence with error values

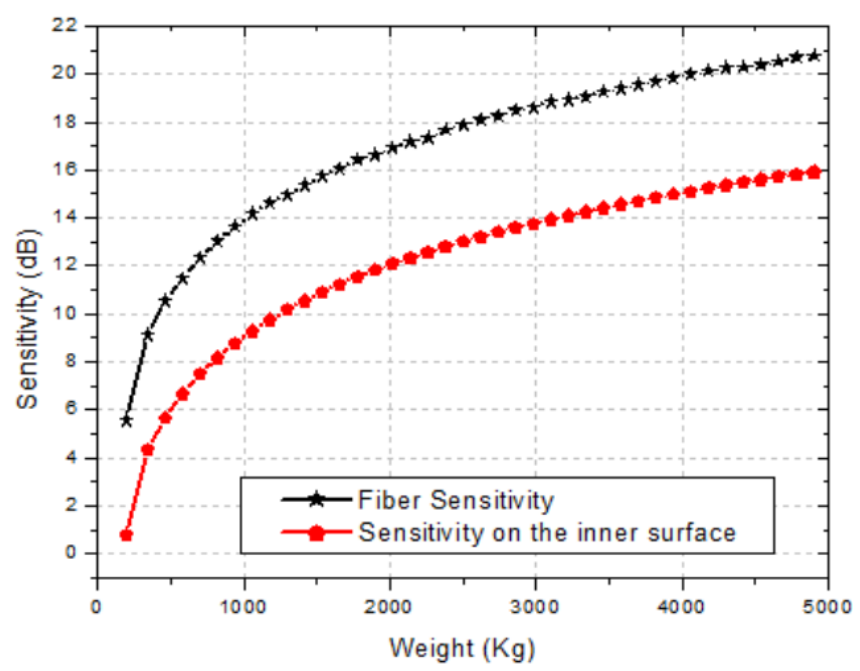

Figure 10. The sensitivity of the core of the fiber and on the apparent surface of the system 


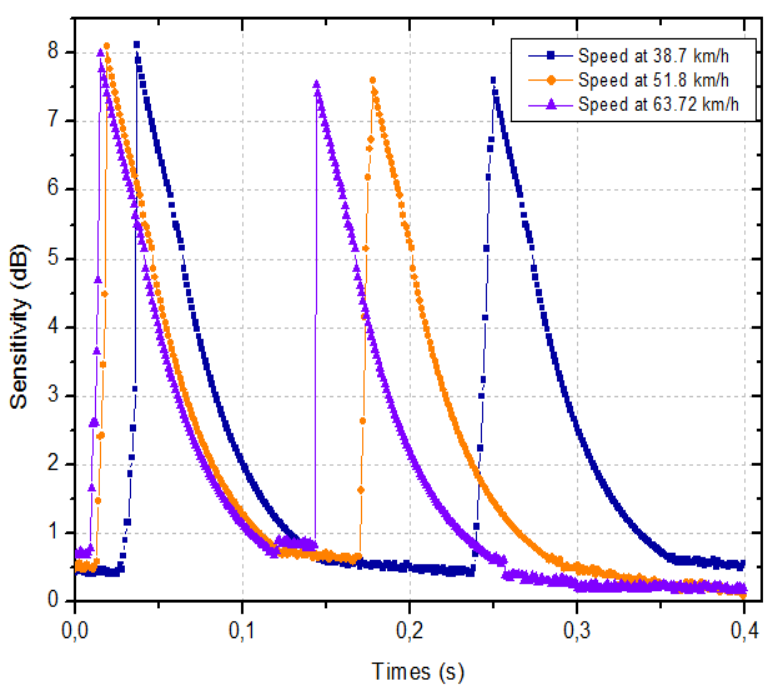

Figure 11. Vehicle A: weight (820 kg+ (130/70)kg ds temp: $\mathbf{2 . 3 m}$

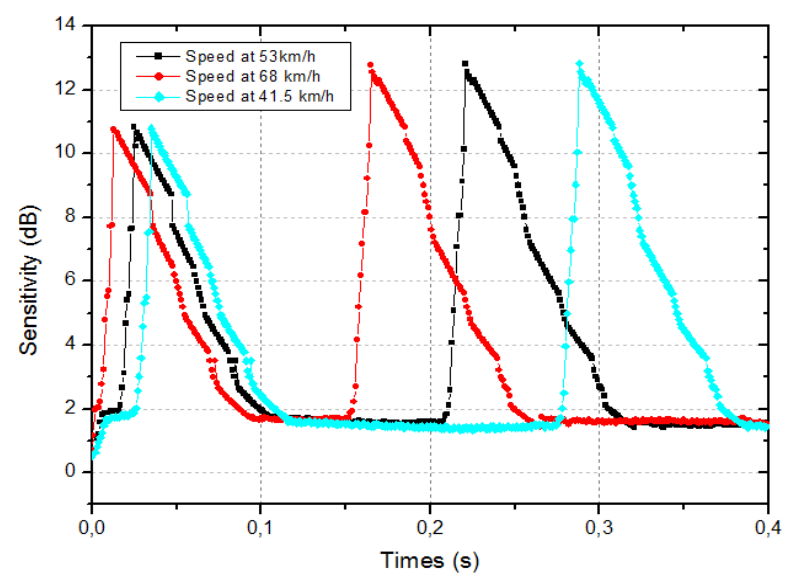

Figure 12. Vehicle $B$ : weight $=1700 \mathrm{~kg} ; \mathrm{D} \_\mathrm{Em}=\mathbf{2 . 9 m}$

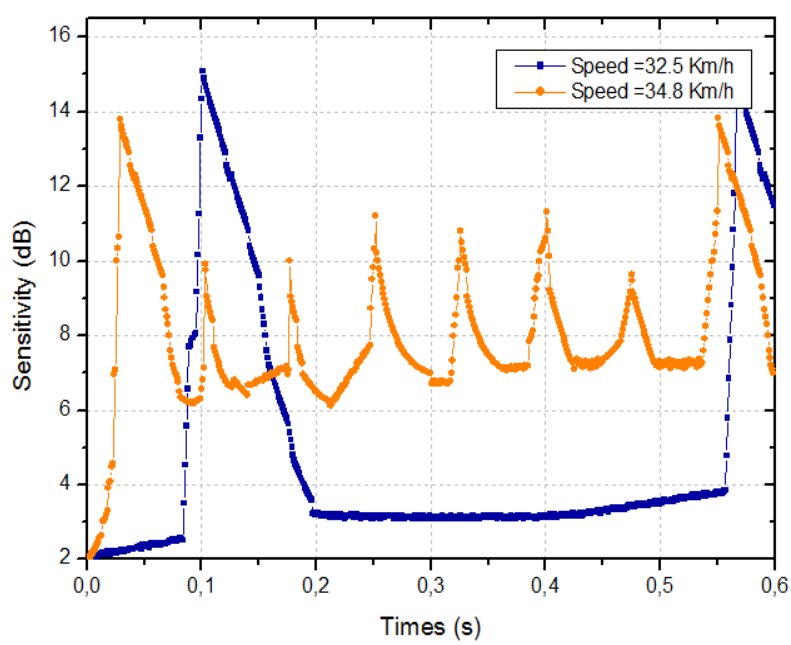

Figure 13. Vehicle C (speed:32.5km/h) and vehicle $D(s p e e d: 34.8 \mathrm{~km} / \mathrm{h}$ )
Advances in the IoT and monitoring systems have gone hand in hand. Indeed, IoT products for military applications use many benefits from novel technologies. The results obtained from our system showed satisfactory values for the detection and classification of intruders. We confirm that the pressure detection error rate from the wavelength is negligible; it is of the order of $0.05 \mathrm{~kg} / \mathrm{cm}^{2}$. The error rate from the weight calculation from the pressure was approximately $12 \mathrm{~kg}$, and the error rate from the wheelbase distance calculation was approximately $0.012 \mathrm{~m}$. This value is marginal and could not distort the decision. In fact, this error range is tolerable, as long as it does not cause interference between the different types in the table listed in our system's database (Table 1). In future work, it will be meaningful to implement a classification algorithm based on machine learning. This will allow us to expand the database stored within the system until it can cover all types, including those that will be started in the future.

\section{ACKNOWLEDGMENT}

This work is part of a Tunisian-South African scientific research project. We thank the Ministry of Higher Education and Scientific Research of Tunisia for supporting this research.

\section{REFERENCES:}

1. ITU-T Rec. Y.2060 (06_2012) Overview of the Internet of things, 2012

2. Otles, S., \& Sakalli, A., 2019. Industry 4.0: The Smart Factory of the Future in Beverage Industry. In Production and Management of Beverages (pp. 439-469). Elsevier

3. N. M. Theune et al. "Investigation of Stator Coil and Lead Temperatures on High Voltage inside Large Power Generators via use of Fiber Bragg Gratings", 2002 IEEE, VOL. 5, 2002.

4. Xiaojun et al. , "IOT- Based Air Pollution Monitoring and Forecasting System", International Conference on Computer and Computational Sciences, VOL 4, 2015.

5. J.C. Zhao et al. "The Study and Application of the 1OT Technology in Agriculture", 2010 IEEE, VOL. 4, 2010.

6. H. Khlaifi et al. "Improvement of Target Discrimination for Monitoring Area Application by a New Proposed Network Design of WSN", 2018 IEEE IWCMC, VOL 6, 2018.

7. M.H.Jridi, H Khlaifi et al. "Targets Classification Based on Multi-sensor Data Fusion and Supervised Learning for Surveillance Application", Wireless Personal Communications, 2019.

8. H. Khlaifi et al., "Routing Protocols for A Border Monitoring Application", International Conference on Internet of Things, Embedded Systems and Communications (IINTEC), 2019.

9. A.Zrelli, H. Khlaifi et al., "Application of damage detection for bridge health monitoring", International Conference on Internet of Things, Embedded Systems and Communications (IINTEC), 2017.

\section{v. CONCLUSION}


10. M.H.Jridi, H.Khlaifi et al., "Coverage and Connectivity of WSN Models for Health Open-Pit Mines Monitoring", IEEE IWCMC, 2018.

11. Y. Imai and T. Hokazono, "Fluorescence-based temperature sensing using erbium-doped optical fibers with $1.48 \mu \mathrm{m}$ pumping," Opt. Rev. 4(1), 117-120, 1997.

12. Othonos, "Fiber bragg gratings", Rev Sci. Instrum. vol68 no12 4309-4341, 1997.

13. Rao et al. "Polymer Packaged Fiber Grating Pressure Sensor with Enhanced Sensitivity", International Journal of Optoelectronic Engineering, VOL 5 NO.4, 2014.

14. Othonos and K. Kalli, "Fiber Bragg Gratings: Fundamentals and Applications inTelecommunications and sensing", Artech House Inc., Boston, London, 1999

15. H.Khlaifi et al. (2021), "Optical Fiber Sensors in Border Detection Application: Temperature, Strain and Pressure Distinguished Detection using Fiber Bragg Grating and Fluorescence Intensity Ratio", Optik - International Journal for Light and Electron Optics, 2021.

16. H. J. Sheng, M.Y. Fu, T.C. Chen, W.F. Liu, and S.S. Bor, A Lateral Pressure Sensor Using a Fiber Bragg Grating, IEEE Photonics Tech. Lett.,vol. 16,pp.1146-1148, Apr. 2004.

17. Amira et al (2016). Monitoring of temperature in distributed optical sensor: Raman and Brillouin spectrum, Optik 127 (2016) 4162-4166,

18. Bouyahi et al. (2016), Modeling the Brillouin spectrum by measurement of the distributed strain and tempera-ture, Opt Quant Electron 48, pp 102112 .

19. Frank Meinl et al. (2017), An Experimental High Performance Radar System for Highly Automated Driving, IEEE MTT-S International Conference on Microwaves for Intelligent Mobility (ICMIM),pp 1-4.

20. Le Zheng et al. (2019) Radar and Communication Coexistence: An Overview: A Review of Recent Methods," in IEEE Signal Processing Magazine, vol. 36 , no. 5, pp. 85-99. 